\title{
transmission des vibrations dues au trafic routier
}

\author{
par \\ Y. Guillard \\ Laboratoire Régional des Ponts et Chaussées, Strasbourg
}

RESUME - Cette action de recherche relative aux vibrations dues au trafic routier a été entreprise dans le but de définir une méthode d'estimation prévisionnelle. L'étude a été scindée en trois parties concernant respectivement le transfert chaussée-sol, la propagation des ondes dans le sol et la transmission sol-bâtiment. Seules les deux premières phases sont abordées ici.

En ce qui concerne la fonction de transfert chaussée-sol, il a été mis au point un modèle qui consiste à assimiler la chaussée lorsqu'elle est excitée verticalement à une fondation circulaire de rayon apparent do, ce dernier étant estimé par ajustement des impédances théorique et expérimentale. Des mesures ont été faites en différents endroits au moyen du
vibreur lourd du L.C.P.C.

Les essais sur la propagation des ondes consistent à exciter le sol par des impulsions et à enregistrer les vibrations résultantes en surface. Pour les vibrations verticales, il a été estimé un coefficient d'amortissement à partir d'un modèle qui tient compte à la fois d'une atténuation en $1 / \sqrt{r}$ et d'une atténuation en $1 / r^{2}$. Le coefficient ainsi évalué varie à peu près linéairement avec la fréquence.

\section{INTRODUCTION}

Le passage d'un véhicule crée des vibrations dans la chaussée et dans les bâtiments

adjacents. Ces vibrations ont deux origines :

1 - Les variations des forces de contact entre les roues d'un véhicule et la surface de la chaussée engendrent dans cette dernière des vibrations qui sont transmises aux bâtiments par le sol.

2 - Hormis les ondes sonores dues au moteur, l'air transmet des ondes de pression résultant de la dimension, de la forme et de la vitesse du véhicule.

Le présent rapport traite uniquement des vibrations solidiennes. Elles se situent généralement entre quelques $\mathrm{Hz}$ et une cinquantaine de $\mathrm{Hz}$ avec une prédominance vers $15-20 \mathrm{~Hz}$. Elles ont un double effet :

- de gêne ressentie par l'homme

- de dégradation dans les bâtiments, le plus souvent par effet indirect (tassement du sol).

Pour étudier ce problème, dont le but est de mettre au point une méthode prévisionnelle, il y a lieu de scinder le système chausséebâtiment en trois parties qui sont le système chaussée-sol, le milieu de propagation et le système sol-structure.

Nous avons jusqu'à maintenant étudié les deux premières parties séparément,

objet des paragraphes ci-dessoús.

\section{TRANSFERT CHAUSSEE-SOL}

Une étude antérieure avait permis de situer les différents paramètres régissant les vibrations dues au trafic routier, du moins au niveau de la chaussée. Il s'agit par ordre d'importance :

- de I'état de I'uni de la chaussée

- du sol support

- de la charge des essieux du véhicule

- de la vitesse du véhicule

Les vibrations les plus importantes étant évidemment obtenues lors du passage du véhicule sur des obstacles du type nid de poule, c'est donc ce cas que nous tenterons de simuler. 
Nous assimilerons pour celà la chaussée à une fondation circulaire de rayon apparent $\mathrm{d}_{\mathrm{o}}$ oscillant verticalement.

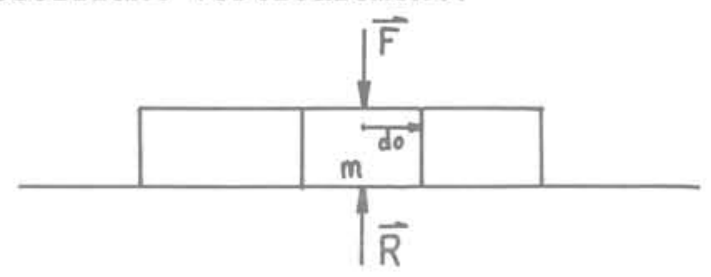

L'évaluation du rayon $d_{0}$ de $l a$ fondation qui aurait le même effet que la chaussée lorsque cette dernière est sollicitée se fera par un ajustement des impédances mesurée et calculée depuis le modèle décrit ci-dessous :

\section{Calcul de l'impédance d'une fondation}

Nous admettrons :

1) que les pressions sous la fondation sont réparties uniformément.

2) que les effets de bord verticaux sont nuls.

Dans ces conditions, si z est le déplacement de l'aire de contact de la fondation avec le sol, m la masse de la fondation, F la force appliquée, $\mathrm{R}$ la réaction du sol, alors :

$m \ddot{z}+R \exp (i \omega t)=F \exp (i(\omega t+\phi))$

$z$ peut être calculé à partir des résultats de REISSNER qui a évalué la réponse dynamique d'une semelle vibrante sur un milieu semiinfini, homogène, isotrope et élastique. Cette semelle est représentée par une surface sans masse qui produit une pression verticale distribuée uniformément sur une aire circulaire de rayon do.

Par intégration de la solution de LAMB, il obtient au centre de la semelle un déplacement de la forme :

$$
z=\frac{R \exp (i \omega t)}{E d_{0}}\left(F_{1}\left(a_{0}\right)+i F_{2}\left(a_{0}\right)\right)
$$

où

$$
E=\text { module de cisaillement du sol }
$$

$$
\begin{aligned}
& a_{0}=\omega d_{0} / v_{s} \\
& \omega=2 \pi f=\text { vitesse angulaire de } \\
& \text { l'excitation }
\end{aligned}
$$$$
\mathrm{V}_{\mathrm{S}}=\text { vitesse des ondes transversales }
$$
dans le sol

$F_{1}, F_{2}=$ fonctions de REISSNER dépendant de $a_{0}$ et du coefficient de Poisson $\nu$ du sol sous la fondation pour $\boldsymbol{\nu}=0,5, F_{1}$ et $F_{2}$ ont la forme suivante :

$$
\begin{aligned}
& F_{1}=-0,130+0,0536 a_{0}{ }^{2}-0,0078 a_{0}{ }^{4}+\ldots \\
& F_{2}=0,0545 J_{1}\left(\frac{1}{2}, 047 a_{0}\right)+J_{0}\left(1,047 a_{0}\right)+0,047 a_{0}
\end{aligned}
$$

avec $J_{0}\left(a_{0}\right)$ et $J_{1}\left(a_{0}\right)$ fonctions de Bessel

Compte tenu de ces résultats et de l'équation (1), il vient, sous la fondation :

$$
\left\{\begin{array}{l}
z=\frac{F}{E d_{0}} \sqrt{\frac{F_{1}{ }^{2}+F{ }^{2}}{\left(1+b a_{0}{ }^{2} F_{1}\right)^{2}+\left(b a_{0}{ }^{2} F_{2}\right)^{2}}} \\
\operatorname{tg} \phi=-\frac{F_{2}}{F_{1}+b a_{0}{ }^{2}\left(F_{1}{ }^{2}+F_{2}{ }^{2}\right)}
\end{array}\right.
$$

ou, si vo est la vitesse du déplacement de la fondation, $Z_{0}$ l'impédance du système, $\mathrm{b}=\mathrm{m} / \rho \mathrm{d}_{0}{ }^{3}, \rho \mathrm{la}$ densité $\mathrm{du}$ sol

$$
\left|z_{0}\right|=\left|\frac{F}{v_{0}}\right|=\rho v_{s} d_{0}^{2} \frac{1}{a_{0}} \sqrt{\frac{\left(1+b a_{0}{ }^{2} F_{1}\right)^{2}+\left(b a_{0}{ }^{2} F_{2}\right)^{2}}{F_{1}{ }^{2}+F_{2}{ }^{2}}}
$$

Parmi les paramètres nécessaires au calcul de cette impédance, 3 ne sont pas connus a priori, nous les déterminerons par ajustement des courbes théorique et expérimentale, il s'agit de :

- do, rayon apparent

- b, paramètre adimensionnel

- $V_{S}$, vitesse des ondes transversales, différente de celle que nous pourrions mesurer à proximité de la chaussée puisque sous cette dernière le sol a généralement été remanié et compacté.

Mesure de l'impédance

Cette mesure consistait à évaluer l'impédance du système chaussée-sol lorsqu'il est excité verticalement.

Le matériel utilisé à cet effet est le vibreur lourd. C'est un excitateur qui soumet la structure étudiée à des efforts verticaux de façon sinusoldale ou en large bande entre une dizaine et une centaine de $\mathrm{Hz}$. Les efforts fournis sont comparables à ceux produits par le jumelage d'un essieu de 13 tonnes. Le contact avec la chaussée se fait par l'intermédiaire d'une plaque métallique de $50 \mathrm{~cm}$ de diamètre laquelle est équipée de capteurs de force et de géophones. Des essais sur 3 sites ont conduits aux résultats de la figure 1 . 


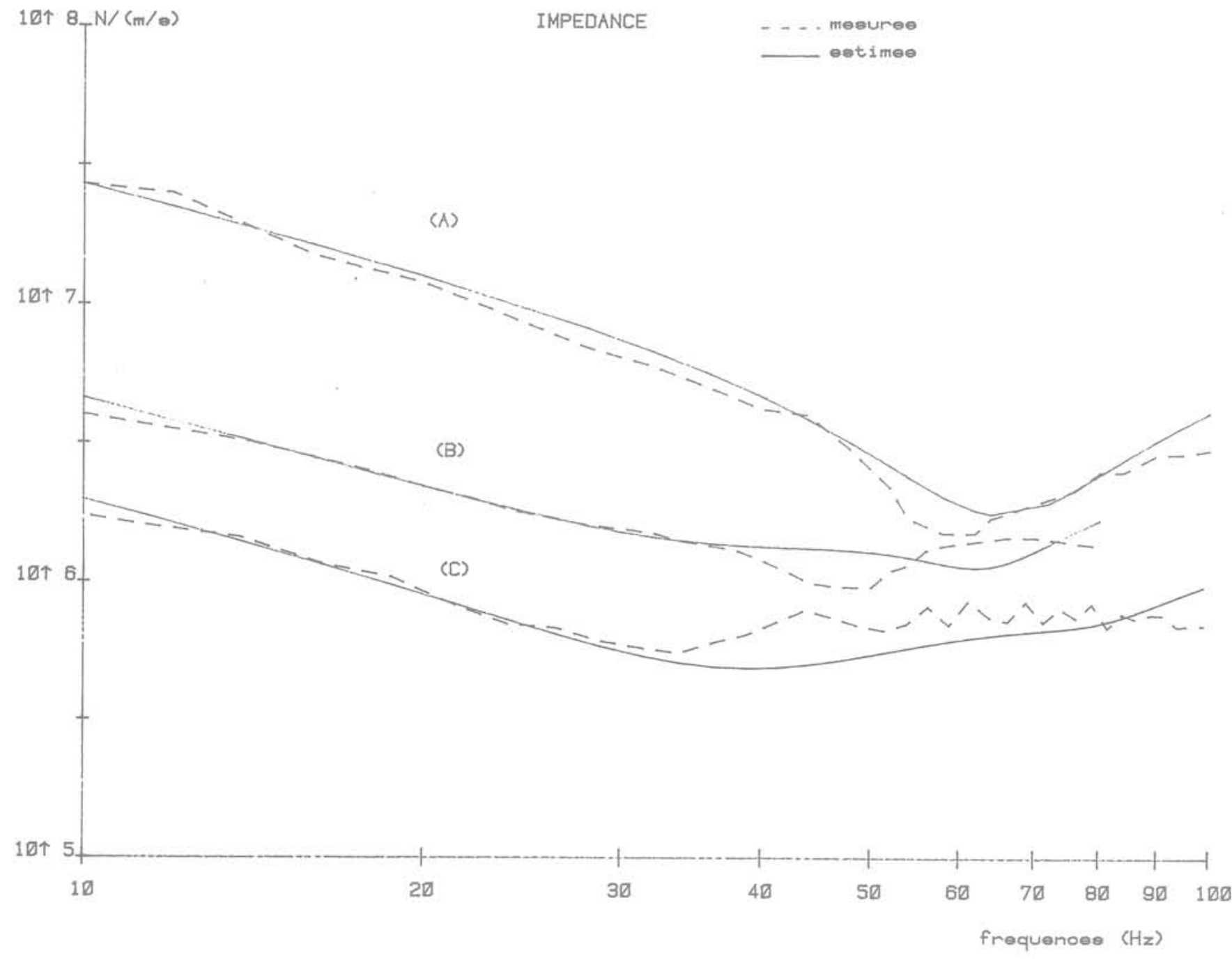

Figure 1 : comparaison des impédances mesurées et ajustées pour différents sites

(A) chaussée souple sur calcaire effrité

$\left(d_{0}=0,6 \mathrm{~m} ; \mathrm{b}=24 ; \mathrm{V}_{\mathrm{S}}=450 \mathrm{~m} / \mathrm{s}\right)$

(B) chaussée de béton sur du sable (de Fontainebleau)

$\left(d_{0}=0,95 \mathrm{~m} ; \mathrm{b}=2,4 ; V_{\mathrm{S}}=150 \mathrm{~m} / \mathrm{s}\right)$

(C) chaussée souple sur sable argileux

$\left(d_{0}=0,6 ; b=4,1 ; V_{S}=125 \mathrm{~m} / \mathrm{s}\right)$ 
Les valeurs de $d_{O}, b$ et $V_{S}$ ont été estimées au moyen d'un programme réalisé sur minicalculateur. Les courbes expérimentales et ajustées montrent qu'il existe une relativement bonne corrélation, essentiellement aux basses fréquences (entre 10 et $40 \mathrm{~Hz}$ ), domaine des vibrations dues au trafic routier.

Un rayon beaucoup plus faibles $(15 \mathrm{~cm}$ ) a été obtenu sur le $3 e$ site en imposant comme vitesse des ondes transversales celle mesurée $(250 \mathrm{~m} / \mathrm{s})$ sur le sol adjacent. Dans ce cas la source pouvait être considérée comme ponctuelle.

\section{PROPAGATION DES ONDES DANS LE SOL}

A partir de la théorie établie pour un milieu semi-infini, homogène, isotrope et élastique, il est possible de calculer, connaissant les efforts injectés en un point de la surface les composantes verticale $(W(t))$ et horizontale $(Q(t))$ des vibrations en fonction de la distance à ce point.

Selon B. PRANGE, elles peuvent être mises sous la forme(l'excitation est supposée ponctuelle)

$W(t)=(R / E \lambda r) W^{*} \exp \left(i\left(\omega t+\phi_{W}\right)\right)$

$Q(t)=(R / E \lambda r) Q^{*} \exp (i(\omega t+\phi q))$

avec

$\lambda r=1$ ongueur des ondes de Rayleigh et $W^{*}, Q^{*}, \phi_{\mathrm{W}}, \phi_{\mathrm{q}}$ modules et phases des expressions $\mathrm{c}^{2}$-dessous

W $\mathrm{E} \lambda \mathrm{r} / \mathrm{R}=$

$\frac{w_{0}}{d 1 / 2}[\cos (-2 \pi d-\pi / 4)+i \sin (-2 \pi d-\pi / 4)]$

$+\frac{w_{1}}{d^{2}}\left[\cos \left(-2 \pi k d^{1 / 2}+\pi / 2\right)+i \sin \left(-2 \pi k d^{1 / 2}+\pi / 2\right)\right]$

$+\frac{W_{2}}{d^{2}}\left[\cos \left(-2 \pi h d^{1 / 2}+\pi / 2\right)+i \sin \left(-2 \pi h d^{1 / 2}+\pi / 2\right)\right]$

$Q E \lambda R / R=$

$\frac{Q_{0}}{d 1 / 2}[\cos (-2 \pi d-3 \pi / 4)+i \sin (-2 \pi d-3 \pi / 4)]$

$+\frac{Q_{1}}{d^{2}}\left[\cos \left(-2 \pi k d^{1 / 2}+i \sin \left(-2 \pi k d^{1 / 2}\right)\right]\right.$

$+\frac{Q_{2}}{d^{2}}\left[\cos \left(-2 \pi h d^{1 / 2}+\pi / 2\right)+i \sin \left(-2 \pi h d^{1 / 2}+\pi / 2\right)\right]$ $e t$

$\mathrm{W}_{\mathrm{O}}=$ $\frac{-k(1-h) \frac{1}{2}}{8\left[(2-k)-\sqrt{(1-h)(1-k)}-\frac{1}{2} \sqrt{\frac{1-k}{1-h}-\frac{1}{2}} \sqrt{\frac{1-h}{1-k}}\right]}$

$w_{1}=(1-1) / 2 k^{1 / 2}$

$W_{2}=1 / 4^{2} h \frac{1}{2}(21-1)^{2}$

$Q_{0}=\frac{-(2-k-2 \sqrt{(1-k)(1-h)})}{8\left[(2-k)-\sqrt{(1-h)(1-k)}-1 / 2 \sqrt{\frac{1-k}{1-h}-1 / 2} \sqrt{\frac{1-h}{1-k}}\right]}$

$Q_{1}=\sqrt{(1-1) / k}$

$Q_{2}=\sqrt{1^{3}-1^{4}} /(21-1)^{3}$

$\mathrm{h}=\left(\mathrm{V}_{\mathrm{R}} / \mathrm{V}_{\mathrm{p}}\right)^{2}$

$\mathrm{k}=\left(\mathrm{V}_{\mathrm{R}} / \mathrm{v}_{\mathrm{S}}\right)^{2}$

$1=\left(V_{s} / v_{p}\right)^{2}$

$V_{R}=$ vitesse des ondes de Rayleigh

$V_{p}=$ vitesse des ondes longitudinales

$V_{S}=$ vitesse des ondes transversales

Des termes en $1 / d \frac{1}{2}$ et $1 / d^{2}$ apparaissent, ils caractérisent respectivement les ondes de Rayleigh et de volume.

Ces résultats se rapportent à un milieu élastique, pour introduire la notion de visco-élasticité il faudra prendre en compte un terme en $\exp (-\alpha d), \alpha$ étant le coefficient d'amortissement des ondes dans le sol.

Résultats des mesures

Le but des mesures était de vérifier ce modèle et d'estimer le coefficient d'amortissement en fonction de la fréquence pour différents sols.

Elles consistaient à relever, en fonction de la distance à la source les vibrations produites par une impulsion. L'excitateur était la dynaplaque, appareil qui peut être schématisé par une plaque de $40 \mathrm{~cm}$ de diamètre posée sur le sol et équipée de ressorts sur lesquels tombe une masse. Les efforts engendrés sont également comparables à ceux produits par le jumelage d'un essieu.

Les signaux étaient captés selon les 3 axes par des accéléromètres piézoélectriques puis enregistrés sur bande magnétique pour être exploités ultérieurement en laboratoire.

La dynaplaque était généralement positionnée sur d'anciennes chaussées ou des voies de desserte et l'atténuation mesurée perpendiculairement à cette voie. Il a été vérifié que la force d'excitation ne variait pas entre le début et la fin des essais. 
Actuellement, seules les mesures selon les axes verticaux ont été exploitées

Une vingtaine d'impulsions étant enregistrées par point de mesure, l'exploitation a consisté à faire une sommation dans le domaine temporel suivi d'une analyse spectrale (voir exemple figure 2).

Pour chaque fréquence, il a été calculé un coefficient d'amortissement à partir d'un ajustement du modèle précédent sur les résultats expérimentaux dont on trouvera quelques exemples sur la figure 3 . Un lissage du type $\left(1 / d \frac{1}{2}\right) \exp (-\alpha d)$ conduit à une moins bonne corrélation aux faibles distances.

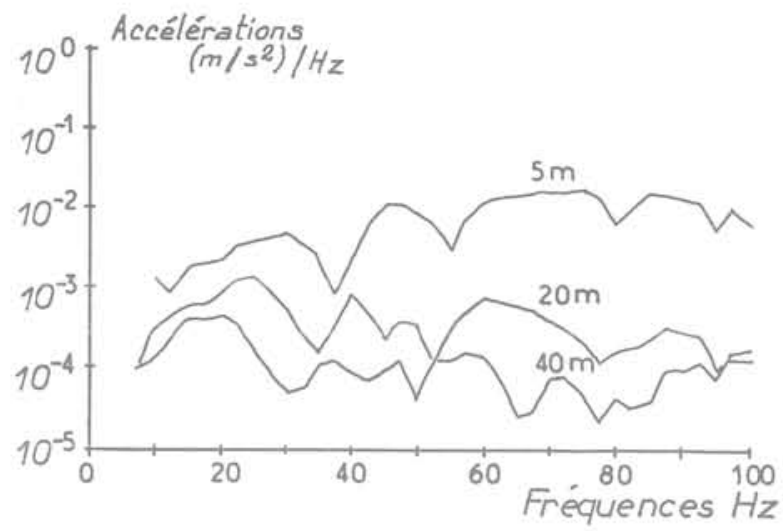

On remarquera sur la figure 4 que le

coefficient d'amortissement crôt à peu près linéairement avec la fréquence.

Figure 2 : Spectres d'accélération en fonction de la distance obtenus sur un sable argileux $(\Delta f=2,5 \mathrm{~Hz})$
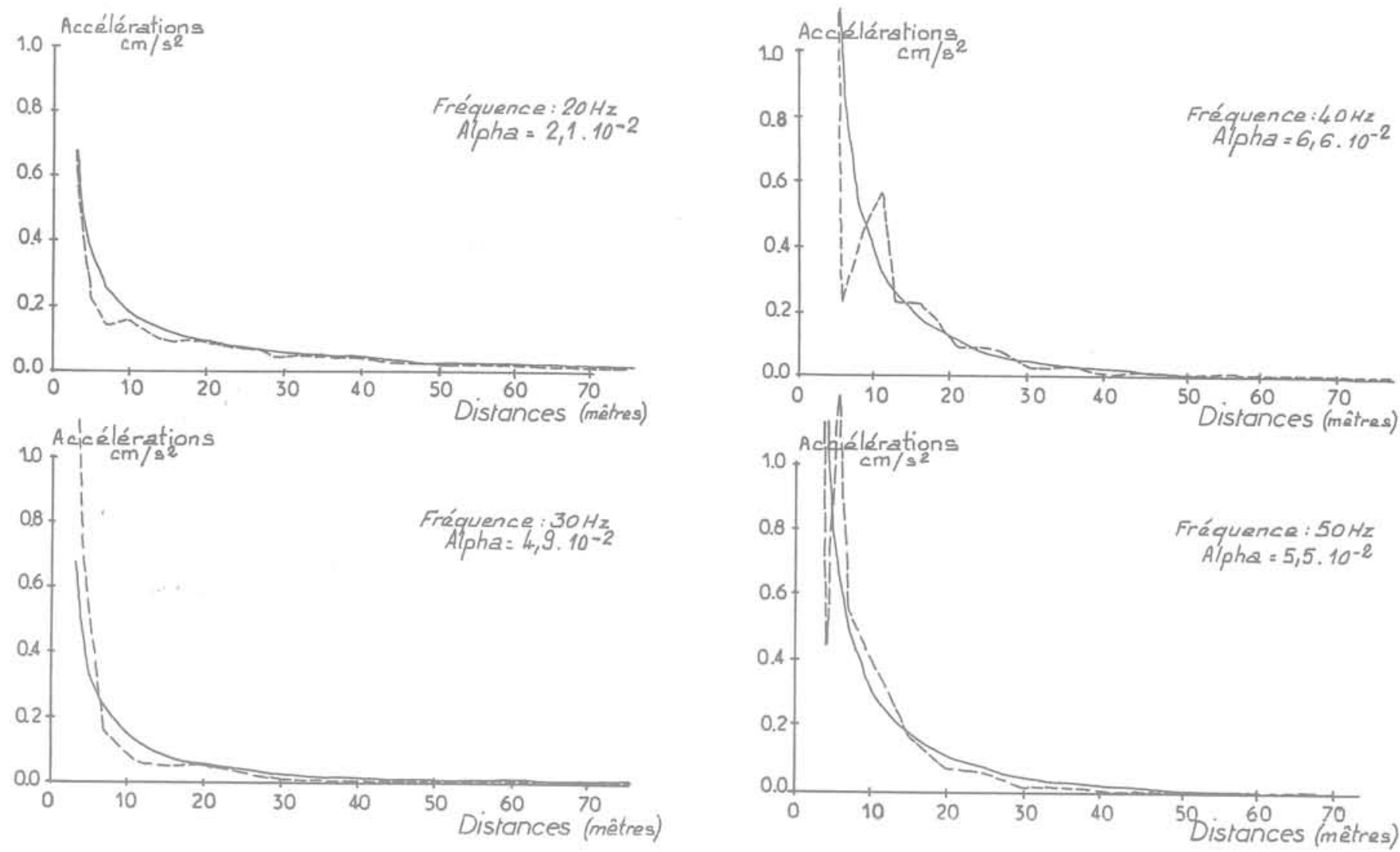

Figure 3 : Atténuation des ondes en fonction de la distance pour quelques fréquences sur du sable argileux -lissage du type $\left(w_{0} / d^{1} / 2+w_{1} / d^{2}\right) \exp (-\alpha d)$ 

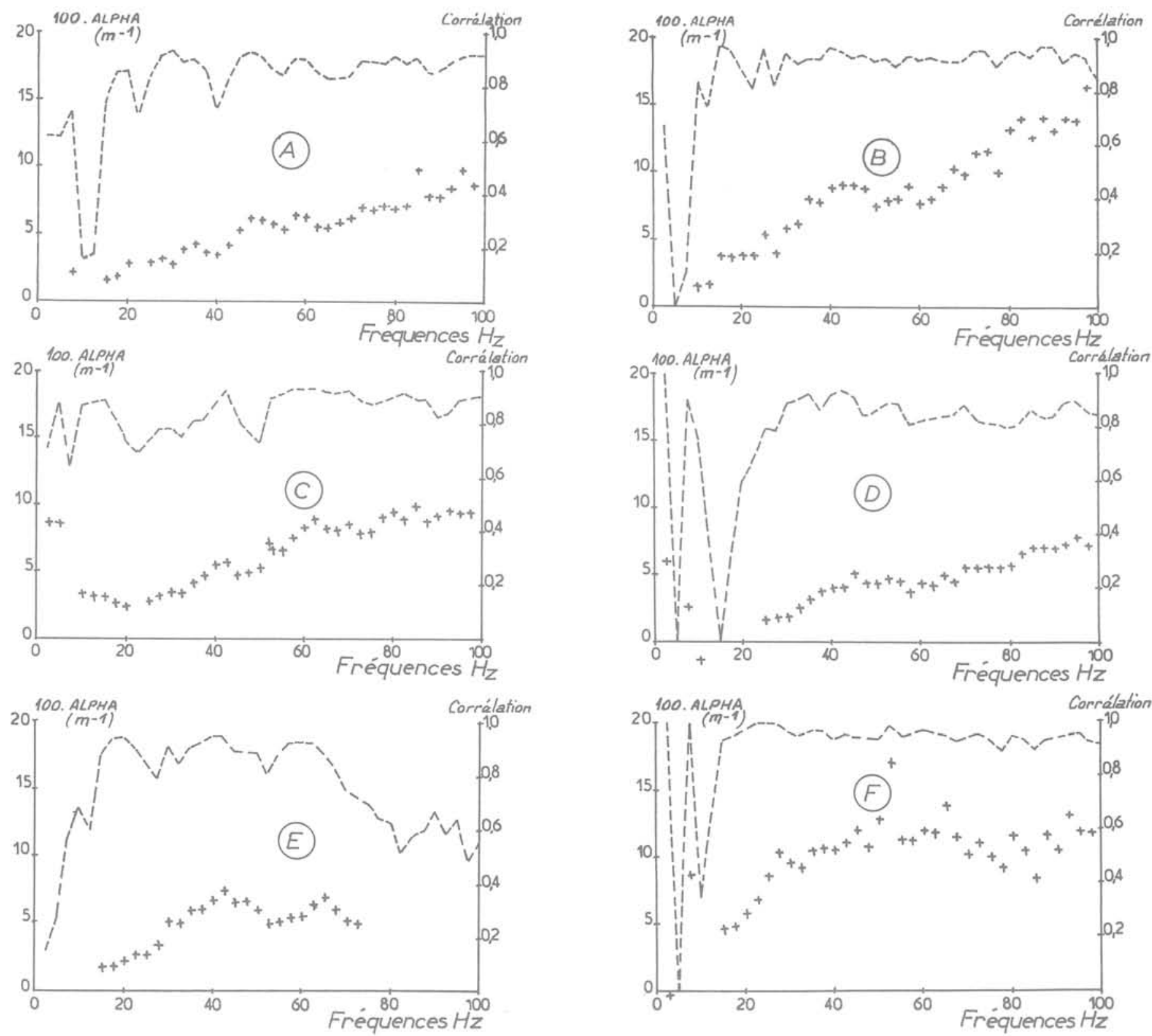

Figure 4 : Coefficient d'amortissement en fonction de la fréquence
(A) remblai
(B) gravier saturé
(C) gravier
(D) sable
(E) sable argileux
(F) argile

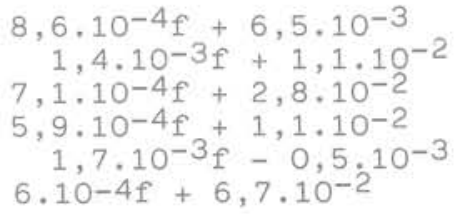




\section{CONCLUSION}

Le but de cette étude était de vérifier différents modèles par des essais in situ. L'un se rapportant au transfert chaussée-sol, l'autre à la propagation des ondes dans le sol.

Lorsque la chaussée est excitée verticalement en un point, cas le plus défavorable d'un point de vue niveau vibratoire, elle peut être assimilée à une fondation circulaire de rayon apparent $\mathrm{d}_{0}$. Des essais ont conduit pour une chaussée rigide à un rayon de 1'ordre du mètre et pour deux chaussées souples à des rayons voisins de $60 \mathrm{~cm}$.

Le coefficient d'amortissement des ondes de surface selon $1^{\prime}$ axe vertical estimé sur plusieurs sites à partir d'un modèle qui tient compte d'une atténuation en $1 / \sqrt{\mathrm{d}}$ et d'une atténuation en $1 / d^{2}$ crồt à peu près linéairement avec la fréquence. La pente, selon le cas, varie entre $10^{-4}$ et $10^{-3}$.

Une combinaison de ces deux résultats devrait conduire à un modèle qui, connaissant la force appliquée à la chaussée, permette d'estimer les niveaux vibratoires du sol en I'absence de bâtiment.

\section{REFERENCES}

Bycroft (1956)

Forced vibrations of a rigid circular plate on a semi-infinite elastic space and on an elastic stratum

Phil. Trans. Roy. Soc., London, Vol. 248, pp $327-368$

B. Prange (1977)

Primary and secondary interferences in wavefields Proceedings of DMSR 77, Karlsruhe 5 - 6 september 1977

Reissner

in Bycroft, op. cit. 